\title{
3D Printed Flowmeter Based on Venturi Effect with Integrated Pressure Sensors ${ }^{+}$
}

\author{
Krzysztof Adamski *, Bartosz Kawa and Rafał Walczak \\ Faculty of Microsystems and photonics, Wroclaw University of Science and Technology, 50-370 Wroclaw, \\ Poland; bartosz.kawa@pwr.edu.pl (B.K.); rafal.walczak@pwr.edu.pl (R.W.) \\ * Correspondence: krzysztof.adamski@pwr.edu.pl; Tel.: +48-71-320-4976 \\ † Presented at the Eurosensors 2018 Conference, Graz, Austria, 9-12 September 2018.
}

Published: 21 December 2018

\begin{abstract}
In this paper we present a 3D printed flow meter based on venturri effect. Dimensions of the microchannels are $800 \mu \mathrm{m}$ for wider and $400 \mu \mathrm{m}$ for thinker channel. Application of different type of sensors was investigated: differential, absolute and digital barometer. Results of measurement of differential pressure and calculation of liquid flow are shown. Presented microfluidics device can be also easy adapted for modular systems. Presented flow meter is the first integration of commercial available sensors and 3D printed microfluidics structure in a single chip.
\end{abstract}

Keywords: 3D printing; integrated sensors; pressure sensor; venturi effect; flow; microfluidics

\section{Introduction}

Flow sensing in microfluidics is important issue. Many of physical effect are applied for flow measurement. Sensor with heat transport [1], utilizing microcantilevers [2], optical detection [3] and based on Venturi effect are widely used. Last of them based on Venturi effect is relatively easy to implement in microfluidic device. Typical microfluidic flowmeters are made from silicon or glass. Structure of the sensor is micromachined in substrates. Next the substrates are bonded together to obtain microfluidic structure. Microengineering of the sensor involves expensive materials (i.e., silicon, borosilicate glass), hazardous liquids (i.e., $\mathrm{KOH}, \mathrm{HF}$ ) and many photolithographic steps. Important issue is also that these technics are time consuming. Also problematic is fabrication of connectors enabling plug of consumer grade sensors (temperature, flow etc.) and flow actuators in pumping system. On the other side polymer sensors (made of PDMS or thermoplastics) require fabrication a forming mold. After forming a structure of channels it is necessary to connect them with other parts of the chip. It limits geometry of the microfluidic structure and complicates technology of the sensor. Therefore new technologies of sensors fabrication [4], microfluidic structures [5] and integrated systems [6] are developed. One of them is 3D printing. 3D printing is one of the technics that can change the world around us [5]. There are many technics of 3D printing but not all of them can be used for fabrication of microfluidic structures and sensors. Selected by us inkjet 3D printing (i3DP) technique enables development of sophisticated microfluidic structures with integrated microfluidics channel with the smallest dimension near to $200 \mu \mathrm{m}$ [7]. Smallest channels are heavy to fabricate due to limited printer resolution and possibility of support material removing. Designing flexibility and truly 3D geometry enables easy integration of sensing electronics element inside a structure of a chip [6]. This feature enable to build smaller and more integrated device. In our previous works it was also proved that inkjet 3D printing technique can be applied for fabrication of lab-on-achip for DNA separation [8], active microfluidics structure as microvalve [9] and integrated siliconglass-polymer systems [10]. Inkjet 3D printing also enables fast and reliable fabrication of modular microfluidics system [11]. 


\section{Measurement Method}

Change of liquid pressure in a tube is a repercussion of Bernoulli law. Flowing liquid moves in a tube called Venturi tube. Tube consist of one fluidic channels with various diameters, first part of them is thicker than second one (Figure 1). Pressure difference (P1 and P2) can be used to calculate flow value (Equation (1)). This effect is also called a venture effect. Thus in order to determine flow the microfluidic structure combined with two pressure sensors innecessary.

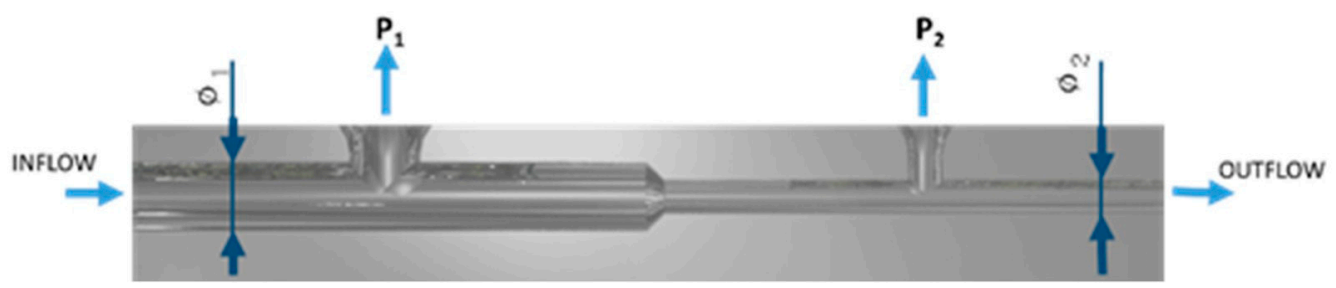

Figure 1. Venturi tube: $\varnothing 1$ input diameter, $\mathrm{P} 1$ pressure measured in input part of the tube, $\mathrm{P} 2$ pressure in output part of the tube with Ø2 output diameter.

$$
Q=A_{1} \sqrt{\frac{2\left(p_{1}-p_{2}\right)}{\rho\left(\left(\frac{A_{1}}{A_{2}}\right)^{2}-1\right)}}
$$

Developed flow sensor was designed with Autodesk Inventor Professional 2018 (Autodesk Inc., San Rafael, CA, USA). Inkjet 3D printing system from 3D Systems Projet 3510 (USA) was utilized with XHD printing mode. Single layer thickness was $16 \mu \mathrm{m}$ and planar $(X-Y)$ resolution was $750 \mathrm{dpi}$. Flow meter was printed in parallel direction with final surface rouges of $0.388 \mu \mathrm{m}$ [9]. The flowmeter was designed with connectors for installing various pressure sensors chip inside the structure. Visijet M3 build material and S300 wax support material were used for fabrication. After printing process the support material was removed from structure in post processing treatment carried out a mineral oil bath, detergent cleaning, and ultrasonic cleaning. All of presented here sensors have the same channel dimensions. Larger channel is $800 \mu \mathrm{m}$ in diameter, smaller channel is $400 \mu \mathrm{m}$ in diameter. Length of each part of the tube is $3 \mathrm{~mm}$. Printed and assembled sensor are showed on Figure 2. Three types of pressure sensors were investigated: MPX5004DP (NXP, Eindhoven, Holland) differential pressure sensor, MS5401 (TE connectivity, Schaffhausen Switzerland) absolute pressure sensor and barometric pressure sensor MPL115A2 (NXP, Eindhoven, Holland).
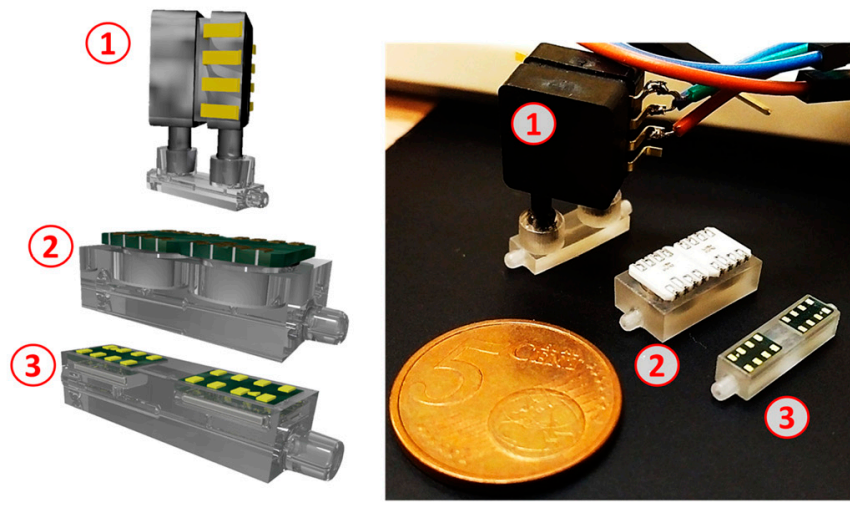

(1)

Flow meter with differential pressure sensor MPX5004

Flow meter with absolute pressure sensor MS401

Flow meter with digital pressure sensor MPL115A2

Figure 2. Different flow meter configurations - left computer design, right-printed sensor assembled with different type of pressure sensor.

MEMS sensors were installed in microfluidic structure and secured for fluid leaking by photo curable polymer glue NORLAN NOA-63 (Thorlabs, Newton, MA, USA). The flow meters have fluidic connectors designed for connection with fluidic tube or modular microfluidics system. An 
example of printed flow sensor connected with modular microfluidic device based on Lego-bricks, described in [11], is shown on Figure 3.

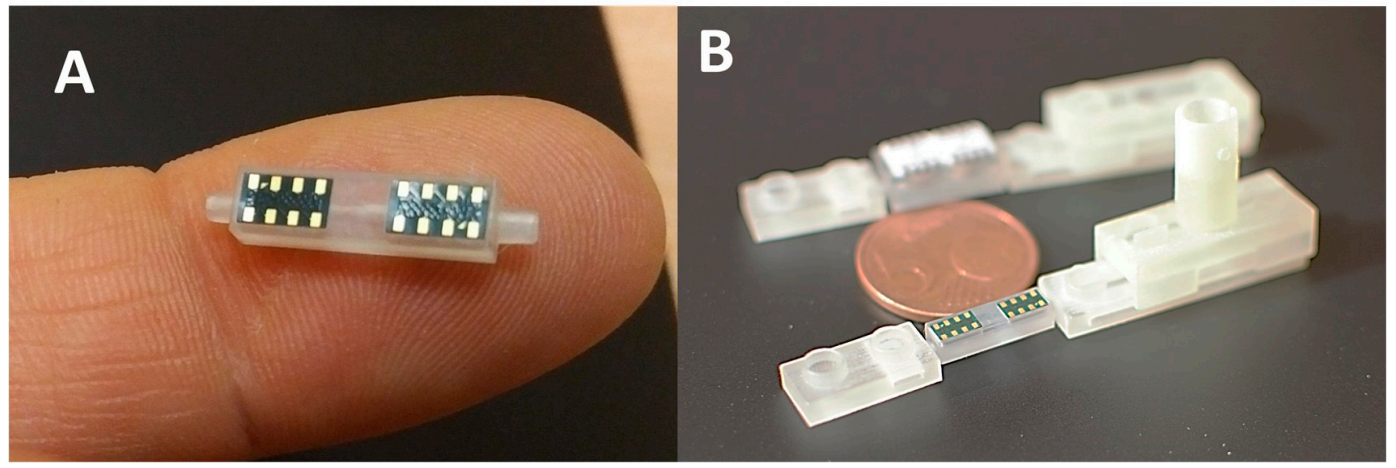

Figure 3. 3D printed flow meter: (A) as standalone part with tubing connectors to a micropump, (B) as a part of modular microfluidic system based on Lego-like brick system.

\section{Measurement Set Up}

Fabricated sensors were tested and characterized in a measurement set-up shown on Figure 4. As external flow generator miniature MP6 micropump (Bartels Microtechnic, Dortmund Germany) with external micropump controller XU-7 were used. Liquid flow was calculated from pumped liquid volume divided by time of pumping. Pumped liquid was water at $22{ }^{\circ} \mathrm{C}$ temperature with density of $0.998 \mathrm{~g} / \mathrm{mm}^{3}$. Pressure signals from sensor (MS401 and MPL115A2) were subtracted and calculated as $\triangle \mathrm{P}$. For differential pressure sensor (MPX5004) this step was unnecessary. Calculated differential pressure was used to calculate liquid flow utilizing Equation (1).

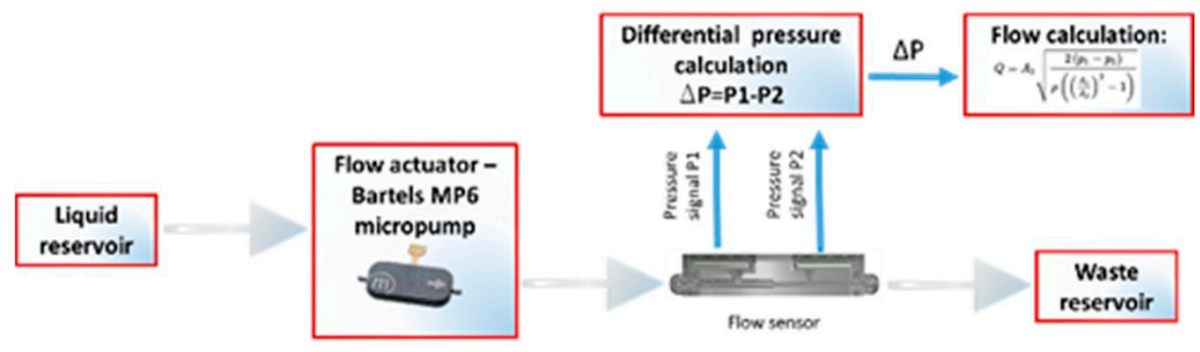

Figure 4. Scheme of the set-up for flow meter characterization.

\section{Results}

Obtained results show that is possible to fabricate Venturi effect flow sensor by application of 3D printing technology and MEMS pressure sensors. Measured MEMS sensors pressure signals were stable and reliable. An example of sensor output characteristic (flow versus differential pressure) for integrated MPX5004DP pressure sensor is presented on Figure 5.

Measured flows-pumped volume vs time (reference method) and by the developed sensor were in good agreement. In case of MPX sensor it was possible to measure water flow in the range of 0-1.4 mL/s (Figure 3a). For constant flow it was also possible to determine concentration of the $\mathrm{C}_{2} \mathrm{H}_{5} \mathrm{OH}$ in water solution (Figure $3 \mathrm{~b}$ ). Flow sensor parameters were stable and reliable. 

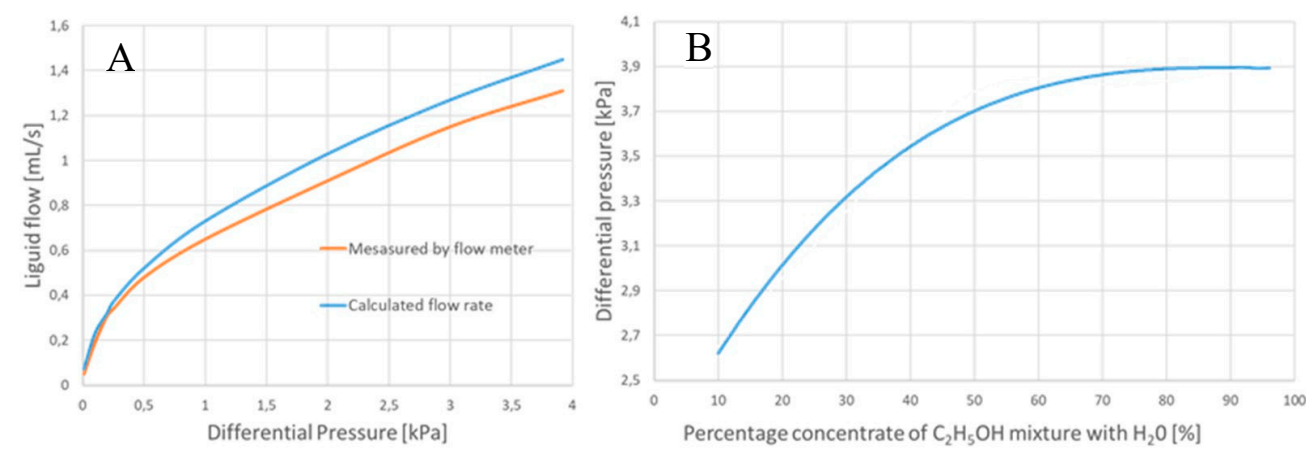

Figure 5. Example of obtained output characteristics for flow meter with integrated MPX5004DP sensor: (A) water flow as function of differential pressure, (B) differential pressure as function of concertation of $\mathrm{C}_{2} \mathrm{H}_{5} \mathrm{OH}$ in water. Solution (mixture flow $=4.8 \mathrm{~cm}^{3} / \mathrm{min}$ ).

\section{Summary}

3D printing enables fast and low-cost fabrication of integrated microfluidic structure as flow meter. In this paper we demonstrated that inkjet 3D printing can be successfully used to developed microfluidic part of the Venturi effect flowmeter combined with MEMS pressure sensors. Measurement range of the flowmeter was in 0-1.4 mL/s range for MPX sensor. Also other two type of pressure sensor were studied. It is worth to mention that ready for work sensor was made from virtual CAD model to existing element in less than $10 \mathrm{~h}$. Thus 3D printing is powerful method enabling microfluidic structures development with short idea-to-element time and great flexibility in geometry of the microstructures.

Acknowledgments: The works are financed by Wroclaw University of Science and Technology by young scientist statuary grant number 0402/0051/17 and statuary grand number 0401/0019/17.

\section{References}

1. Kuo, J.T.W.; Yu, L.; Meng, E. Micromachined Thermal Flow Sensors-A Review. Micromachines 2012, 3, 550573, doi:10.3390/mi3030550.

2. Wang, Y.-H.; Chen, C.-P.; Chang, C.-M.; Lin, C.-P.; Lin, C.-H. MEMS-based gas flow sensors. Microfluid. Nanofluid. 2009, 6, 333-346, doi:10.1007/s10404-008-0383-4.

3. Wang, Y.; Li, Z.; Qin, L.; Chyu, M.K.; Wang, Q.-M. Theoretical and Experimental Studies of a Surface Acoustic Wave Flow Sensor. IEEE Trans. Ultrason. Ferroelectr. Freq. Control 2012, 59, doi:10.1109/TUFFC.2012.2218.

4. Leigh, S.J.; Bradley, R.J.; Purssell, C.P.; Billson, D.R.; Hutchins, D.A. A Simple, Low-Cost Conductive Composite Material for 3D Printing of Electronic Sensors. PLoS ONE 2012, 7, e49365, doi:10.1371/journal.pone.0049365.

5. Waldbaur, A.; Rapp, H.; Länge, K.; Rapp, B.E. Let there be chip-Towards rapid prototyping of microfluidic devices: One-step manufacturing processes. Anal. Methods 2011, 3, 2681-2716, doi:10.1039/C1AY05253E.

6. Adamski, K.; Adamski, J.; Dziuban, J.A.; Walczak, R. Inkjet 3D Printed Miniature Water Turbine Energy Harvester-Flow Meter for Distributed Measurement Systems. Proceedings 2017, 1, 578, doi:10.3390/proceedings1040578.

7. Walczak, R.; Adamski, K. Inkjet 3D printing of microfluidic structures-On the selection of the printer towards printing your own microfluidic chips. J. Micromech. Microeng. 2015, 25, doi:10.1088/09601317/25/8/085013.

8. Walczak, R.; Adamski, K.; Kubicki, W. Inkjet 3D printed chip for capillary gel electrophoresis. Sens. Actuators B Chem. 2018, 261, 474-480.

9. Walczak, R.; Adamski, K.; Lizanets, D. Inkjet 3D printed check microvalve. J. Micromech. Microeng. 2017, 27, doi:10.1088/1361-6439/aa6152. 
10. Krysztof, M.; Grzebyk, T.; Górecka-Drzazga, A.; Adamski, K.; Dziuban, J. Electron optics column for a new MEMS-type transmission electron microscope. Bull. Polish Acad. Sci-Tech. Sci. 2018, 66, 133-137.

11. Walczak, R.; Adamski, K.; Kubicki, W. Configurable on-Chip Gel Electrophoresis in Inkjet 3D Printed Microfluidic Modules. Proceedings 2017, 1, 520, doi:10.3390/proceedings1040520.

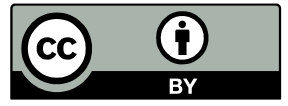

(C) 2018 by the authors. Licensee MDPI, Basel, Switzerland. This article is an open access article distributed under the terms and conditions of the Creative Commons Attribution (CC BY) license (http://creativecommons.org/licenses/by/4.0/). 